\title{
Effectiveness and Safety of Low-dose Rituximab in the treatment of Demyelinating Diseases of the
}

\section{Central Nervous System}

Mahisa mokhtari, MD a; Meysam Abolmaali, MD b; Maziar Emamikhah, MD a; Nafiseh Mohebi, MD a; Jaber

Hatam, MD c; Elaheh Amini, MD a; Parisa Saiyarsarai, PhD d, e; Seyed Amir Hassan Habibi, MD a; Mehdi

Moghaddasi, MD a*

a Department of neurology, Hazrat Rasool hospital, Iran university of medical sciences (IUMS), Tehran, Iran

b Student Research Committee, School of Medicine, Iran University of Medical Sciences (IUMS), Tehran, Iran

c Department of neurosurgery, Hazrat Rasool hospital, Iran university of medical sciences (IUMS), Tehran, Iran

d Department of Pharmacoeconomics and Pharmaceutical Administration, Faculty of Pharmacy, Tehran University of

Medical Sciences (TUMS), Tehran, Iran

e The Institute of Pharmaceutical Sciences (TIPS), Tehran University of Medical Sciences, Tehran, Iran

*Corresponding author contact information:

Mehdi Moghaddasi, MD

Professor of Neurology

Division of Neurology - Hazrat Rasool Hospital

Iran University of Medical Sciences

Niyayesh St, Sattarkhan Ave

Tehran, Iran

Postal code: 1445613131

Phone: +98 2166525331

Fax: +98 2166525331

E-mail: $\underline{\text { moghaddasim@ @otmail.com }}$ 
Total word count of the manuscript including title page, references, and structured abstract: 2942

Running title (less than 40 characters): Low-dose rituximab, safe and effective in MS/NMO

Keywords: Rituximab; Multiple Sclerosis; Neuromyelitis Optica; demyelinating diseases

\section{Abstract}

Background: Rituximab is a monoclonal antibody widely used in the treatment of inflammatory and autoimmune disorders. Despite reports of its effectiveness in the treatment of demyelinating diseases of central nervous system (DDCNS), it is not yet approved for use in these disorders. The aim of this study was to investigate the effectiveness and safety of low dose rituximab in three different subgroups of DDCNS including relapsing-remitting multiple sclerosis (RRMS), secondary-progressive multiple sclerosis (SPMS) and neuromyelitis-optica-spectrum disorders (NMOSD).

Methods: In a prospective cohort study, we monitored expanded-disability-status-scale (EDSS), relapses (new attacks) and serum-IgG levels to assess effectiveness and drug-adverse-events for safety in patients with RRMS, SPMS and NMOSD. These patients were candidates to receive rituximab according to our common practice protocol. We switched patients to rituximab if there was poor response to first line therapies. We follow a low dose protocol in our center (500 mg twice, two weeks apart, repeating every six months) and these patients treated in a 4-year period were assessed retrospectively for evaluation of our protocol's safety and effectiveness.

Results: 99 patients (42 RRMS, 43 SPMS and 14 NMOSD) received rituximab for a range of 12 to 40 months period. New attacks occurred in 8 RRMS (19\%), 10 SPMS (23\%) and 1 NMOSD (7\%) patients. EDSS decreased in RRMS and NMOSD cases. Serum-IgG levels decremented in SPMS and NMOSD patients. Drug-adverse-events happened in two cases. 
Conclusion: In this study, low dose rituximab showed substantial effectiveness in preventing disease progression with a considerably good safety profile. 


\section{Introduction}

Rituximab is a human/mouse chimeric monoclonal antibody agent that acts against the CD20 expressed receptors of B-cells ${ }^{1}$. These cells are playing an important role in the immune response and are thought to be involved in the development of a number of autoimmune diseases including demyelinating disease of the central nervous system (DDCNS) ${ }^{2}$. Multiple sclerosis (MS) and neuromyelitis-optica spectrum disorders (NMOSD), also known as Devic's disease are considered as the two most common DDCNSs in the literature. Although these are two distinct disorders of myelin in central nervous system (CNS), both etiologically and pathophysiologically, they share similar clinical features and treatment strategies. Rituximab has been regarded as the most successful synthetic monoclonal antibody agent used in the treatment of hematolymphoid malignancies ${ }^{3}$. Originally, it was approved for the use in patients with Bcell non-hodgkin's lymphoma. Since then, it has received approval for the treatment of other lymphatic malignancies and a number of inflammatory and autoimmune disorders ${ }^{4-6}$. Currently, it is regarded as a suitable off-label choice drug for the treatment of DDCNS alongside the other disease-modifying therapies including interferon $\beta$, glatiramer acetate, mitoxantrone, natalizumab and alemtuzumab ${ }^{7}$.

Despite previous confirmatory studies on patients with other different diseases, there have been few studies conducted on the safety and effectiveness of rituximab in patients with DDCNS ${ }^{8,9}$. In this regard, the safety and effectiveness of rituximab administration in patients with DDCNS must be confirmed by more comprehensive cohort and trial studies. Furthermore, there is controversy regarding the optimum dosing of rituximab in different protocols. Most studies has chosen the higher dose approach (e.g. $1000 \mathrm{mg}$ on weeks $0,2,24,48 \ldots$ or administered every six months) or started with higher dose and then changed to lower-dose regimen $(500 \mathrm{mg})$ in the subsequent maintenance doses ${ }^{9,10}$. Some has also introduced a minimally effective dose approach (100 mg per dose) repeated usually every six months ${ }^{11}$. The aim of this study was to investigate the safety and effectiveness of low-dose rituximab in 
patients with relapsing-remitting MS (RRMS), secondary progressive MS (SPMS) and NMOSD, a strategy which has been less well-evaluated ${ }^{12,13}$.

\section{Material and Methods}

This study was approved by the institutional review board and ethics committee of Iran University of Medical Sciences, Tehran, Iran; and has been conducted in accordance with the ethical standards of Declaration of Helsinki. Informed consent was obtained from all patients before administration of rituximab.

Based on our protocol for the management of patients with MS spectrum and NMOSD, rituximab is not considered as the first-choice disease modifying drug (DMD) and is prescribed in cases with poor response to first line therapeutic agents (e.g. interferon $\beta$ or Dimethyl fumarate for MS). According to our common practice in our center, the patients receive $500 \mathrm{mg}$ rituximab per dose, ("Zytux" ARYOGEN Co, Tehran, Iran) by slow intravenous infusion over a 4-hour period. We administered rituximab in two separate courses, two-weeks apart and these two doses were repeated every 6 months to restrain the disease from further attacks. As premedication, patients received methyl prednisolone (125 mg intravenous infusion), chlorpheniramine (10 mg intravenous stat) and acetaminophen (1000 mg oral) 30 minutes before the administration of rituximab, to inhibit any possible inflammatory reactions.

In this retrospective cohort study, we collected and analyzed demographics, clinical, radiological and lab data of patients with DDCNS treated in a single tertiary center, the hospital which first author is affiliated to, between 2016 and 2019. Based on expert diagnosis, patients were divided into three subgroups: RRMS, SPMS and NMOSD. MS and NMOSD were diagnosed based on McDonald's criteria 2010 and International consensus diagnostic criteria for neuromyelitis optica spectrum disorders $2015^{14}$,

${ }^{15}$, respectively. All clinical and paraclinical data of patients during a close follow-up of at least 12 
months (after receiving 4 infusions of rituximab) was collected. There was no patient with an antimyelin oligodendrocyte glycoprotein (anti-MOG) demyelinating disease spectrum diagnosis in our data set. During the follow-up period, neurological evaluation and laboratory tests were routinely performed every six months for all patients after the first course of rituximab. Patients were assessed by brain, cervical and thoracic contrast-MRIs annually to investigate probable new lesions, using a 1.5 Tesla scanner. Serum immunoglobulin G (IgG) level were also measured every six months. Neurological disability status was assessed by the expanded disability status scale (EDSS) and any change in EDSS during the follow-up was assessed for patients in each group. Moreover, number of new attacks during treatment (relapse) and any infusion-related adverse effects of rituximab were also documented. All collected data were analyzed by The International Business Machines Corporation (IBM) Statistical Package for the Social Sciences (SPSS), version 22.0 and $p$ values were calculated with the Wilcoxon signed-rank test. Meanwhile, all participants provided written informed consent for both treatment with rituximab and participating in this study.

\section{Results}

Between February 2016 and December 2019, a total number of 99 patients with RRMS, SPMS and NMOSD whose therapy was changes to rituximab, were selected prospectively. 42 patients were diagnosed with RRMS (42.5\%), 43 with SPMS (43.4\%) and 14 with NMOSD (14.1\%). The mean age of patients was $39.5 \pm 10.7$ years (between 18 and 66). 67 patients $(67.7 \%$ ) were female. Groups were similar in their age and sex average and no significant different was found between groups' demographics $(p>0.05)$. Disease duration were ranged between 2 to 30 (mean 8.97) years and the mean follow-up for rituximab-therapy was $20.4 \pm 7.9$ months (ranged 12 to 40 ). The number of attacks during treatment with rituximab were 8 (19\%), 10 (23.2\%) and $1(7 \%)$ among patients with RRMS, 
SPMS and NMOSD, respectively. Among the 19 patients who had experienced attacks during the follow-up period, 13 had one episode, four patients had two episodes and two patients had experienced three episodes of attacks. The last EDSS was subtracted from the baseline EDSS for each patient. Mean EDSS variability had decreased in RRMS $(-0.32, \mathrm{P}=0.06)$ and NMOSD $(-0.57, \mathrm{P}=0.004)$ and had a slight increase among patients with SPMS $(+0.19, \mathrm{P}=0.23)$. New lesions on T2-weighted MRI and Gadolinium enhancing lesions were found in 9 and 4 cases respectively, during follow-up MRI (Table 1). Serum IgG level had a declining trend in $13.9 \%$ and $10 \%$ of patients with SPMS and NMOSD, respectively. None of patients with RRMS had serum IgG decrement during follow-up. There was no opportunistic infection in patients with low serum IgG.

\section{Adverse drug effect}

During 818 administered doses of rituximab, only two cases had developed a drug adverse effect during or after rituximab infusion. These adverse effects included flushing and thrombocytopenia in two SPMS patients during and after rituximab administration, respectively. In the first case, a 40-year-old man developed flushing 30 minutes after starting the administration of first dose of rituximab. There were no hives, itching, shortness of breath or hemodynamic changes. Infusion rate declined to half and the patient received the rest of dose without any further complications. The second case, a 37-year-old woman, diagnosed with thrombocytopenia after second dose of rituximab during her routine tests. Treatment was continued and she received the two subsequent doses six months after her first thrombocytopenic event. After the $4^{\text {th }}$ course of rituximab administration, platelets counts dropped to $11.5 \times 10^{9} / \mathrm{L}$ but returned to normal range during next four-months close follow-up without further treatments. 


\section{Discussion}

Despite some variability in the outcome of three sub-types of DDCNS, overall results showed a favorable effectiveness of low-dose rituximab in patients with various forms of DDCNS. Considering the results of our study, $80.8 \%$ of patients experienced a complete disease control without relapses, all of them had suffered treatment failure with other medications in the past. D'Amico et al published a similar study of their experience in administration of rituximab in patients with DDCNS in Italy, in which $65 \%$ of their patients had no evidence of disease activity. They used two courses of rituximab two weeks apart (1000 mg per dosing for adults and $500 \mathrm{mg}$ for pediatric patients) and decided next courses administration timing based on CD19 and CD20 levels. Smaller sample size (seventeen patients) with more proportion of them being NMOSD ( $23 \%$ vs. $14 \%$ in our study) may explain the lower efficacy of rituximab in their investigation ${ }^{2}$.

The interesting point regarding our findings was the non-inferiority of low-dose rituximab regimen compared to higher usual dosing in all three DDCNS groups. Many previous studies who implemented $1000 \mathrm{mg}$ dosing have reported similar or even higher relapse rates in both MS and NMOSD patients ${ }^{2,9}$, 13.

Due to financial problems we were not able to check CD19 and CD20 in all patients, but we used total serum IgG level as an indirect marker of severe B cell depletion and to diagnose patients at the risk for infections secondary to immunosuppression. Our experience shows, close following of these markers are not necessary to ensure effectiveness and safety of the rituximab therapy. This may further rationalize the implementation of low-dose regimen in similar situations, when the routine monitoring of CD19 and CD20 is not available. Besides safety, our results showed that the efficacy of low-dose treatment is noninferior to high dose protocol and there was no infection related complications in our patients. Although the reliability of $\mathrm{IgG}$ monitoring merits further investigations, we recommend routine monitoring of total 
serum IgG in similar situations, as it is a much more available and inexpensive laboratory test compared to flow cytometry. In similar studies, follow up through CD19 and CD 20 monitoring neither reached higher disease control nor faced less adverse events compared to our present results $9,10,13$.

Patients with SPMS had poorer clinical response to treatment and less disease activity control (77\% compared to $81 \%$ in RRMS and $93 \%$ in NMOSD). Moreover, patients with SPMS had the least improvement after rituximab-therapy based on the changes in EDSS score and our only cases with drug adverse events were among patients with SPMS. This is in corroboration with similar studies, which showed less disability improvement in progressive MS patients after rituximab therapy ${ }^{9}$. The higher effectiveness of rituximab in disease-relapse-control, although is biased by small number of patients (one relapse in 14 NMOSD patients), is supported by those studies which used rituximab as first-line DMD for NMOSD and have reported significant relapse control and reduction in EDSS as well as appropriate tolerability 16,17 .

Among the patients with new attack in our study, ambulation deficit was seen in $47.5 \%$ and most of them had active lesion and deficit in pyramidal system (Table 2). This may reflect a selection bias; the patients with more disabling symptoms were selected to receive rituximab as the DMD.

Eventually, we should emphasize that the administration of rituximab as a DMD in DDCNS including MS and NMOSD is still off-label and there is controversy regarding optimum dosage and regimens. The supporting evidence for its use in MS is the recent approval of similar anti-CD20 monoclonal antibody, ocrelizumab, which has shown substantial effectiveness and acceptable safety profile, but is not still as widely available as rituximab and is much more expensive than rituximab at present time ${ }^{18}$.

\section{Conclusion}


Low dose rituximab showed favorable effectiveness in terms of reducing the attack frequencies and improving EDSS between the follow-up intervals among patients with DDCNS. Drug adverse events were negligible overall. SPMS had the least favorable response to rituximab administration among the three main DDCNS. More studies are required to investigate the optimum dosage, regimen protocol and follow up strategies.

\section{Disclosures}

Funding sources: This research did not receive any specific grant from funding agencies in the public, commercial, or not-for-profit sectors.

Declarations of conflicts of interest: none.

Acknowledgement: None.

Data Availability Statement:

The data that support the findings of this study are available from the corresponding author upon reasonable request.

\section{References}

1. Kang J, Kim SY, Vallejo D, et al. Multifaceted assessment of rituximab biosimilarity: The impact of glycan microheterogeneity on Fc function. Eur J Pharm Biopharm. 2020;146: 111-124.

2. D'Amico E, Zanghi A, Chisari CG, et al. Effectiveness and safety of Rituximab in demyelinating diseases spectrum: An Italian experience. Mult Scler Relat Disord. 2019;27: 324-326.

3. Buettner R. Predictive Biomarkers in Lung Cancer. In: Badve S, Kumar GL, eds. Predictive Biomarkers in Oncology: Applications in Precision Medicine. Cham: Springer International Publishing, 2019: 403-409.

4. Jurczak W, Moreira I, Kanakasetty GB, et al. Rituximab biosimilar and reference rituximab in patients with previously untreated advanced follicular lymphoma (ASSIST-FL): primary results from a confirmatory phase 3, double-blind, randomised, controlled study. Lancet Haematol. 2017;4: e350-e361.

5. Honce JM, Nair KV, Sillau S, et al. Rituximab vs placebo induction prior to glatiramer acetate monotherapy in multiple sclerosis. Neurology. 2019;92: e723-e732. 
6. De D, Bishnoi A, Handa S, Mahapatra T, Mahajan R. Effectiveness and safety analysis of rituximab in 146 Indian pemphigus patients: A retrospective single-center review of up to 68 months follow-up. Indian J Dermatol Venereol Leprol. 2020;86: 39-44.

7. McDonell J, Costello K, Laurson-Doube J, et al. World Health Organization Essential Medicines List: Multiple sclerosis disease-modifying therapies application. Mult Scler. 2020: 1352458519898340.

8. Spelman T, Frisell T, Piehl F, Hillert J. Comparative effectiveness of rituximab relative to IFN-beta or glatiramer acetate in relapsing-remitting MS from the Swedish MS registry. Mult Scler. 2018;24: 1087-1095.

9. Scotti B, Disanto G, Sacco R, Guigli M, Zecca C, Gobbi C. Effectiveness and safety of Rituximab in multiple sclerosis: an observational study from Southern Switzerland. PLoS One. 2018;13: e0197415.

10. Hawker K, O'Connor P, Freedman MS, et al. Rituximab in patients with primary progressive multiple sclerosis: results of a randomized double-blind placebo-controlled multicenter trial. Annals of neurology.

2009;66: 460-471.

11. Nielsen AS, Miravalle A, Langer-Gould A, Cooper J, Edwards KR, Kinkel RP. Maximally tolerated versus minimally effective dose: the case of rituximab in multiple sclerosis. Multiple Sclerosis Journal. 2012;18: 377.

12. Salzer J, Svenningsson R, Alping P, et al. Rituximab in multiple sclerosis: a retrospective observational study on safety and efficacy. Neurology. 2016;87: 2074-2081.

13. Greenberg BM, Graves D, Remington G, et al. Rituximab dosing and monitoring strategies in neuromyelitis optica patients: creating strategies for therapeutic success. Multiple Sclerosis Journal. 2012;18: 1022-1026.

14. Thompson AJ, Banwell BL, Barkhof F, et al. Diagnosis of multiple sclerosis: 2017 revisions of the McDonald criteria. Lancet Neurol. 2018;17: 162-173.

15. Wingerchuk DM, Banwell B, Bennett JL, et al. International consensus diagnostic criteria for neuromyelitis optica spectrum disorders. Neurology. 2015;85: 177-189.

16. Zéphir $\mathrm{H}$, Bernard-Valnet R, Lebrun C, et al. Rituximab as first-line therapy in neuromyelitis optica: efficiency and tolerability. Journal of neurology. 2015;262: 2329-2335.

17. Ciron J, Audoin B, Bourre B, et al. Recommendations for the use of Rituximab in neuromyelitis optica spectrum disorders. Revue neurologique. 2018;174: 255-264.

18. McCool R, Wilson $\mathrm{K}$, Arber $\mathrm{M}$, et al. Systematic review and network meta-analysis comparing ocrelizumab with other treatments for relapsing multiple sclerosis. Multiple sclerosis and related disorders. 2019;29: 55-61. 
Table 1. Patients characteristics at first course of Rituximab administration and during follow-up.

\begin{tabular}{|c|c|c|c|}
\hline Features & RRMS $^{+}$ & SPMS $^{\ddagger}$ & NMOSD $^{\S}$ \\
\hline No. of patients, (\%) & $42,(42.5)$ & $43,(43.4)$ & $14,(14.1)$ \\
\hline \multicolumn{4}{|l|}{ Age at presentation (years) } \\
\hline Mean & 34.1 & 43.5 & 43 \\
\hline Range & $18-66$ & $27-60$ & $26-62$ \\
\hline \multicolumn{4}{|l|}{ Gender, No. (\%) } \\
\hline Male & $14,(33.3)$ & $13,(30.2)$ & $5,(35.7)$ \\
\hline Female & $28,(66.7)$ & $30,(69.8)$ & $9,(64.3)$ \\
\hline Mean disease duration (years) & 6.7 & 12.2 & 6 \\
\hline Mean follow-up period (months) & 17.3 & 22.2 & 24.3 \\
\hline $\begin{array}{l}\text { Patients with clinical attacks } \\
\text { (proportion of that specific DDCNS , \%) }\end{array}$ & $8(19)$ & $10(23)$ & $1(7)$ \\
\hline New T2-weighted MRI lesions & 4 & 4 & 1 \\
\hline Gadolinium enhancing lesion & 2 & 2 & 0 \\
\hline Drug reaction & 0 & 2 & 0 \\
\hline EDSS ๆ variability & $\begin{array}{c}-0.32 \pm 1.1 \\
(P=0.06)\end{array}$ & $\begin{array}{l}+0.19 \pm 1 \\
(P=0.23)\end{array}$ & $\begin{array}{l}-0.57 \pm 0.6 \\
(P=0.004)\end{array}$ \\
\hline
\end{tabular}

†) RRMS: Relapsing-remitting multiple sclerosis; ¥) SPMS: Secondary-progressive multiple sclerosis;

§) NMOSD: neuromyelitis optica spectrum disorder; ף) EDSS: Expanded Disability Status Scale 
Table 2. Characteristics of patients experienced new attack during follow-up

\begin{tabular}{|c|c|c|c|c|}
\hline No. & Age, Sex & Type & Localization of the lesion & Symptoms \\
\hline 1 & 42 , female & SPMS $^{+}$ & Pyramidal & Ambulation deficit \\
\hline 2 & 34 , male & RRMS & Pyramidal \& Sensory & $\begin{array}{c}\text { Lower limbs weakness \& } \\
\text { paresthesia }\end{array}$ \\
\hline 3 & 35 , female & RRMS & Cerebellum, Paraventricular \& Cord & Ambulation deficit \\
\hline 4 & 53 , female & SPMS & Cord & Paraparesis \\
\hline 5 & 29 , male & SPMS & Pyramidal & Ambulation deficit \\
\hline 6 & 42 , female & NMOSD $\S$ & Pyramidal & Ambulation deficit \\
\hline 7 & 42 , female & SPMS & Pyramidal & Hemiparesis \\
\hline 8 & 37 , female & SPMS & Pyramidal \& Sensory & Hemiparesis \\
\hline 9 & 39 , male & SPMS & Periventricular & Hemiparesis \& Paresthesia \\
\hline 10 & 38 , female & SPMS & Pyramidal & Hemiparesis \\
\hline 11 & 32 , female & RRMS & Pyramidal & Ambulation deficit \\
\hline 12 & 43 , female & RRMS & Periventricular & Ambulation deficit \\
\hline 13 & 27 , female & SPMS & Pyramidal & Hemiparesis \\
\hline 14 & 29 , female & RRMS & Brain stem & Trigeminal neuritis \\
\hline 15 & 48 , female & RRMS & Pyramidal & Ambulation deficit \\
\hline 16 & 30 , female & RRMS & Cord & $\begin{array}{c}\text { Paraparesis \& Sphincteric } \\
\text { disorder }\end{array}$ \\
\hline 17 & 44 , male & SPMS & Pyramidal & Ambulation deficit \\
\hline 18 & 29 , female & SPMS & Pyramidal & Ambulation deficit \\
\hline 19 & 20 , female & RRMS & Pyramidal, Optic nerve \& Cerebellum & Hemiparesis \& Blurred vision \\
\hline
\end{tabular}

†) SPMS: Secondary-progressive multiple sclerosis; ¥) RRMS: Relapsing-remitting multiple sclerosis;

§) NMOSD: neuromyelitis optica spectrum disorder 